\title{
The prevalence of CT-defined low skeletal muscle mass in patients with metastatic cancer: a cross-sectional multicenter French study (the SCAN study)
}

\author{
Bruno Raynard ${ }^{1}\left[\right.$. Frederic Pigneur ${ }^{2} \cdot$ Mario Di Palma $^{3} \cdot$ Elise Deluche $^{4} \cdot$ François Goldwasser $^{5}$
}

Received: 17 March 2021 / Accepted: 27 September 2021 / Published online: 3 December 2021

(c) The Author(s) 2021

\begin{abstract}
Background Cachexia, characterized by involuntary muscle mass loss, negatively impacts survival outcomes, treatment tolerability, and functionality in cancer patients. However, there is a limited appreciation of the true prevalence of low muscle mass due to inconsistent diagnostic methods and limited oncologist awareness.

Methods Twenty-nine French healthcare establishments participated in this cross-sectional study, recruiting patients with those metastatic cancers most frequently encountered in routine practice (colon, breast, kidney, lung, prostate). The primary outcome was low skeletal muscle mass prevalence, as diagnosed by estimating the skeletal mass index (SMI) in the middle of the third-lumbar vertebrae (L3) level via computed tomography (CT). Other objectives included an evaluation of nutritional management, physical activity, and toxicities related to ongoing treatment.

Results Seven hundred sixty-six patients (49.9\% males) were enrolled with a mean age of 65.0 years. Low muscle mass prevalence was $69.1 \%$. Only one-third of patients with low skeletal muscle mass were receiving nutritional counselling and only $28.4 \%$ were under nutritional management (oral supplements, enteral or parenteral nutrition). Physicians highly underdiagnosed those patients identified with low skeletal muscle mass, as defined by the primary objective, by $74.3 \%$ and $44.9 \%$ in obese and non-obese patients, respectively. Multivariate analyses revealed a lower risk of low skeletal muscle mass for females (OR: $0.22, P<0.01)$ and those without brain metastasis (OR: $0.34, P<0.01)$. Low skeletal muscle mass patients were more likely to have delayed treatment administration due to toxicity $(11.9 \%$ versus $6.8 \%, P=0.04)$.

Conclusions There is a critical need to raise awareness of low skeletal muscle mass diagnosis among oncologists, and for improvements in nutritional management and physical therapies of cancer patients to curb potential cachexia. This calls for cross-disciplinary collaborations among oncologists, nutritionists, physiotherapists, and radiologists.
\end{abstract}

Keywords Cachexia $\cdot$ Low muscle mass $\cdot$ Skeletal mass index (SMI) $\cdot$ Nutritional support

Bruno Raynard

bruno.raynard@gustaveroussy.fr

1 Gustave-Roussy, 114 Rue Edouard Vaillant, 94800 Villejuif, France

2 Henri Mondor University Hospitals, AP-HP Créteil, France

3 American Hospital of Paris, Neuilly-sur-Seine, France

4 Limoges University Hospital, Limoges, France

5 Cochin Hospital, AP-HP, CARPEM, Paris Descartes University, Paris, France

\section{Introduction}

Cancer cachexia is a complex metabolic syndrome characterized by involuntary muscle loss with or without loss of fat mass, systemic inflammation, and negative protein and energy balance. This condition cannot be completely reversed with conventional nutritional care and leads to progressive functional impairment in cancer patients [1-3]. Cancer cachexia is associated with an increased risk of postoperative complications, increased mortality in the metastatic phase, increased treatment intolerance, longer hospitalizations, and alterations in patient quality of life [4-7]. Deleterious effects increase with severity, delaying cancer management. Early care, prior to $5 \%$ bodyweight loss, is thus recommended [7]. 
The most clinically relevant phenotype of cachexia is sarcopenia. In oncology, the diagnosis of sarcopenia is generally based solely upon the loss of skeletal muscle mass. Dual-energy x-ray absorptiometry (DEXA) is the method of reference for measuring muscle mass [8-10]. More recently, estimations of the skeletal mass index (SMI) at the third-lumbar vertebra (L3) level via computed tomography $(\mathrm{CT})$ have been used to define low muscular mass [11-13]. Although this particular application of CT is not yet routinely implemented in France, it has been proposed as an objective measure for the identification of low skeletal muscle mass among cancer patients [14].

Methods of cachexia diagnosis are variable; Fearon et al. [3] propose three different diagnostic criteria, one of which is defined by CT-defined low muscle mass: (1) $>5 \%$ weight loss in the last 6 months, (2) BMI $<20 \mathrm{~kg} / \mathrm{m}^{2}$ and ongoing weight loss $>2 \%$, or (3) sarcopenia, in particular as defined by low SMI (measured by CT segmentations at the midpoint of the L3 vertebrae, using the thresholds of $<55 \mathrm{~cm}^{2} / \mathrm{m}^{2}$ in males and $<39 \mathrm{~cm}^{2} / \mathrm{m}^{2}$ in females [3]) and ongoing weight loss $>2 \%$. These SMI thresholds have since been endorsed by the European Society for Clinical Nutrition and Metabolism [15].

Precise estimates of low muscle mass prevalence among cancer patients remain scarce and vary widely. As such, the primary objective of this cross-sectional, multi-centric study conducted in France was to evaluate the prevalence of low skeletal muscle mass as measured by L3 cross-sectional CT scans in patients diagnosed with metastatic cancers. Moreover, misdiagnoses by oncologists and nutritional strategies available to cancer patients were explored.

\section{Methods}

\section{Study population and design}

This study was conducted in accordance with the Declaration of Helsinki. All persons provided their informed consent prior to enrollment. The protocol was in line with French data protection regulations (CNIL, approval no. 2066086) and approved by the French ethical research committee, "Le Comité de Protection des Personnes" (CPP), on the 6 July 2017 (approval no. 2017-A01648-45).

This cross-sectional study was conducted within the investigators' oncology practices in 29 public and private hospitals in France, between September and October 2017. Patients were males or females aged $\geq 18$ years, diagnosed with metastatic lung, kidney, colon, breast, or prostate cancer, irrespective of the age of the diagnosis. At the time of inclusion, patients had to be undergoing chemotherapy, targeted therapy, hormonotherapy, or immunotherapy that was initiated since at least one cycle of treatment or for at least 1 month, irrespective of the line of ongoing treatment. Patients had to have a CT scan including an L3 cross-section suitable for SMI evaluation of low muscle mass, performed (for any reason) between 6 weeks before and 4 weeks after inclusion to the study. Patients were excluded if diagnosed with two or more malignant pathologies, if being treated exclusively by radiotherapy, if they had surgery in the previous 30 days, or if they had a concomitant neurological comorbidity, other than cerebral metastasis.

The investigating pair in each of the 29 centers consisted of an oncologist-radiologist duo. An obligatory training presentation was given to investigating radiologists for the measurement of the cross-sectional skeletal muscle area (CSA) using CT. This training was provided by a radiologist member of the SCAN study scientific committee using a standardized approach, in order to minimize the risk of observer error in CSA slice selection and in contouring the borders of the skeletal muscles [16]. Muscle mass was determined using manual segmentation on dedicated posttreatment stations (FujiFilm [Synapse 3D], Siemens [syngo. via version VB10 and VB20], GE Healthcare or Toshiba Medical) as a CSA in the middle of L3 level [17]. It should be noted that the decision to permit the use of different posttreatment stations is supported by data from a study that compared 4 different imagery station software and demonstrated an excellent correlation between readings, with limited inter- and intra-operator variability [18]. The total CSA was measured in square centimeters with a pre-established density threshold within -29 to +150 Hounsfield units (HU; including the external and internal obliques, paraspinal, rectus abdominis, transversus abdominis, and psoas muscles). All CSA were individually normalized for stature, as is conventional for body composition evaluation, resulting in the SMI: SMI $=$ CSA/height ${ }^{2}\left(\mathrm{~cm}^{2} / \mathrm{m}^{2}\right)[16,19]$. These SMI were subsequently evaluated by the investigating radiologist.

Oncologists, who were blinded to the CT scan results, recorded the following information during the inclusion visit: current weight, weight 1 and 6 months ago, height, dietary and nutritional support for each patient, weekly physical activity, food intake assessment at the previous meal using a visual analogue scale (VAS, wherein 10 signifies normal, healthy food intake for the patient) [20], and performance status (PS) scores using the Eastern Cooperative Oncology Group (ECOG) scale of 0 (fully active) to 5 (death) [21]. C-reactive protein (CRP) and serum albumin (SA) data were collected from test results between a month before and a week after the CT scan to assess malnutrition in line with local guidelines [22] and the patients' Glasgow prognostic scores (GPS), categorizing patient prognosis on a scale of 0 (best) to 2 (worst) [23].

Malnutrition severity was defined according to criteria that were in use in routine practice. These criteria were defined by the French national health authorities [22], 
using the thresholds listed below by age group: moderate malnutrition $<70$ years old, $\mathrm{BMI} \leq 18.5$ to $16 \mathrm{~kg} / \mathrm{m}^{2}$ or $\mathrm{SA}<30$ to $20 \mathrm{~g} / \mathrm{L}$, and $\geq 70$ years old, $\mathrm{BMI}<21$ to $18 \mathrm{~kg} /$ $\mathrm{m}^{2}$ or $\mathrm{SA}<35$ to $30 \mathrm{~g} / \mathrm{L}$; and severe malnutrition $<70$ years old, BMI $\leq 16 \mathrm{~kg} / \mathrm{m}^{2}$ or $\mathrm{SA}<20 \mathrm{~g} / \mathrm{L}$, and $\geq 70$ years old, $\mathrm{BMI}<18 \mathrm{~kg} / \mathrm{m}^{2}$ or $\mathrm{SA}<30 \mathrm{~g} / \mathrm{L}$.

Additionally, the oncologist was required to assess for low skeletal muscle mass as per their discretion (no predefined method), while being blinded to the CT results.

\section{Study objectives}

The primary objective was to determine the prevalence of low skeletal muscle mass in patients with metastatic cancer of the lung, kidney, colon, breast, or prostate, by estimating on CT the skeletal muscle index (SMI) in the middle of the L3 level, with cut-off values $<55 \mathrm{~cm}^{2} / \mathrm{m}^{2}$ and $<39 \mathrm{~cm}^{2} / \mathrm{m}^{2}$ indicating low muscle mass in males and females, respectively [3].

Other outcomes included (1) an evaluation of the level of agreement between CT measurements and oncologists' evaluations of low muscle mass (for the total study population, and for obese (body mass index $[\mathrm{BMI}] \geq 30 \mathrm{~kg} / \mathrm{m}^{2}$ ) and non-obese sub-groups $\left(\mathrm{BMI}<30 \mathrm{~kg} / \mathrm{m}^{2}\right)$; (2) a description of nutritional care in cancer patients (proportion of patients receiving nutritional counselling and nutritional management); (3) weekly physical activity of cancer patients (equivalent to $30 \mathrm{~min}$ of walking or cycling on flat terrain, or swimming), and daily hours spent in bed as reported by the patient; and (4) an evaluation of toxicities related to ongoing anti-cancer treatment and their impact on dosage modifications, treatment delays due to toxicity, treatment interruptions, and the occurrence of adverse events $(\mathrm{AE}) \geq$ grade 3 . AEs were graded according to the Common Terminology Criteria for Adverse Events (CTCAE).

Cachexia prevalence in patients with low muscle mass was estimated in an exploratory manner according to each of the three criteria defined by Fearon et al. [3], which were elaborated in the introduction. Multivariate analyses of the relationship between the presence of low muscle mass and patient age, sex, primary tumor site, weight loss, current BMI, serum albumin, PS score, VAS for food intake, and brain metastasis were also performed.

\section{Statistical analyses}

Data management and statistical analyses were performed by Kantar Health (Paris, France), using DAISIE (version 2.4.25 \& 2.4.45) and R i386 3.0.1. Descriptive analyses (means, SD, median, and range) are provided for continuous variables, and percentages for categorical variables. To prevent assumptions on the distribution of data, the nonparametric Wilcoxon signed rank test was used to evaluate the difference in mean for continuous variables, and the $Z$ test was used for comparisons of categorical variables. Logistic regression evaluating the relationship between the presence of low muscle mass and selected exploratory variables is expressed in odds ratios (OR) with $95 \%$ confidence intervals (CI). $P$ values for comparisons between the groups with and without low muscle mass were calculated; $P<0.05$ was considered statistically significant.

\section{Results}

A participant flow diagram for this study is presented in Fig. 1.

\section{Low muscle mass prevalence in cancer patients and their clinical characteristics}

The patients' demographic and disease characteristics are presented in Table 1. Males and females were equally represented in the study, with an average age of $65.0 \pm 11.8$ years; cancer types were colon $(37.1 \%)$, lung $(25.5 \%)$, breast $(22.6 \%)$, kidney $(7.8 \%)$, and prostate $(7.0 \%)$. Average times since diagnosis of the primary tumor were $46.9 \pm 60.7$ months. The majority of patients $(40.5 \%)$ were undergoing their first line of treatment.

CT-defined low skeletal muscle mass was considered to be present for patients whose SMI was $<55 \mathrm{~cm}^{2} / \mathrm{m}^{2}$ in males and $<39 \mathrm{~cm}^{2} / \mathrm{m}^{2}$ in females [3]. In the total population of 766 patients, 529 were assessed as to have low muscle mass, denoting a CT-defined low skeletal muscle mass prevalence of $69.1 \%$.

Low muscle mass was more prevalent in males than females: $83.0 \%(317 / 382)$ versus $55.2 \%(212 / 384)$ $(P<0.01)$; and patients with low muscle mass were older than those without. Low muscle mass was most prevalent in prostate cancer patients $(94.4 \%, 51 / 54)$, compared to only

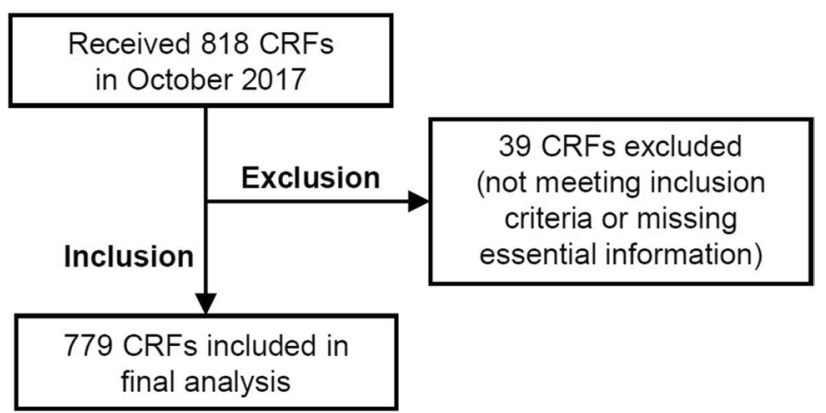

Fig. 1 Flow diagram representing the sample population enrolled in the SCAN study. Of 818 CRFs received in October 2017, 52 were excluded as they did not meet selection criteria or lacked essential information; $766 \mathrm{CRFs}$ were included in the study analysis 
Table 1 Demographic and cancer characteristics of patients with and without low skeletal muscle mass, as assessed by the L3 computed tomography imaging method. A patient has low skeletal muscle mass if the skeletal mass index (SMI) is $<55 \mathrm{~cm}^{2} / \mathrm{m}^{2}$ in men and $<39 \mathrm{~cm}^{2}$ I $\mathrm{m}^{2}$ in women. In the total population of 766 patients, 529 were found to have low skeletal muscle mass, denoting a prevalence of $69.1 \%$. Results are presented by $N(\%)$ or mean \pm SD. NS not significant, $N A$ not applicable. Non-responses are not shown

\begin{tabular}{|c|c|c|c|c|}
\hline & Total patients & Low muscle mass & Unimpaired muscle mass & $P$ \\
\hline $\mathrm{SMI}, \mathrm{cm}^{2} / \mathrm{m}^{2}$ & $n=766$ & $n=529$ & $n=237$ & \\
\hline Mean \pm SD & $43.2 \pm 8.7$ & $40.6 \pm 7.6$ & $49.1 \pm 8.2$ & $P<0.01$ \\
\hline \multirow[t]{2}{*}{ Male, n (\%) } & $n=766$ & $n=529$ & $n=237$ & \\
\hline & $386(49.9)$ & $317(59.9)$ & $65(27.4)$ & $P<0.01$ \\
\hline Age (years) & $n=762$ & $n=527$ & $n=235$ & \\
\hline Mean \pm SD & $65.0 \pm 11.8$ & $66.1 \pm 11.8$ & $62.5 \pm 11.3$ & $P<0.01$ \\
\hline Site of primary tumor, $\mathrm{n}(\%)$ & $n=766$ & $n=529$ & $n=237$ & \\
\hline Colon & $284(37.1)$ & $200(37.8)$ & $84(35.4)$ & NS \\
\hline Lung & $195(25.5)$ & $138(26.1)$ & $57(24.1)$ & NS \\
\hline Breast & $173(22.6)$ & $94(17.8)$ & $79(33.3)$ & $P<0.01$ \\
\hline Kidney & $60(7.8)$ & $46(8.7)$ & $14(5.9)$ & NS \\
\hline Prostate & $54(7.0)$ & $51(9.6)$ & $3(1.3)$ & $P<0.01$ \\
\hline Time since diagnosis of primary cancer (months) & $n=762$ & $n=529$ & $n=237$ & \\
\hline Median (range) & $23.2(0-400.0)$ & $20.8(0-396.0)$ & $28.1(1.0-400.0)$ & NS \\
\hline Time since diagnosis of metastatic disease (months) & $n=756$ & $n=524$ & $n=232$ & \\
\hline Median (range) & $14.5(0-285.0)$ & $13.6(0-285.0)$ & $17.5(0-160.0)$ & NS \\
\hline Main metastatic sites, n (\%) & $n=766$ & $n=529$ & $n=237$ & \\
\hline Hepatic & $323(42.2)$ & $224(42.3)$ & $99(41.8)$ & NS \\
\hline Pulmonary & $310(40.5)$ & $208(39.3)$ & $102(43.0)$ & NS \\
\hline Lymph nodes & $275(35.9)$ & $198(37.4)$ & $77(32.5)$ & NS \\
\hline Bones & $259(33.8)$ & $178(33.6)$ & $81(34.2)$ & NS \\
\hline Peritoneal & $90(11.7)$ & $64(12.1)$ & $26(11.0)$ & NS \\
\hline Cerebral & $81(10.6)$ & $67(12.7)$ & $14(5.9)$ & $P<0.01$ \\
\hline Treatment line, $n(\%)$ & $n=766$ & $n=529$ & $n=237$ & \\
\hline First line of treatment & $310(40.5)$ & $207(39.1)$ & $103(43.5)$ & NS \\
\hline Second line of treatment & $217(28.3)$ & $154(29.1)$ & $63(26.6)$ & NS \\
\hline Third or higher & $208(27.2)$ & $144(27.2)$ & $64(27.0)$ & NS \\
\hline Non-responses & $31(4.0)$ & $24(4.5)$ & $7(3.0)$ & NS \\
\hline Ongoing therapies, $\mathrm{n}(\%)$ & $n=766$ & $n=529$ & $n=237$ & \\
\hline Chemotherapy & $530(69.2)$ & $375(70.9)$ & $155(65.4)$ & NS \\
\hline Hormonotherapy & $72(9.4)$ & $48(9.1)$ & $24(10.1)$ & NS \\
\hline Targeted therapy & $305(39.8)$ & $192(36.3)$ & $113(47.7)$ & $P<0.01$ \\
\hline Immunotherapy & $118(15.4)$ & $89(16.8)$ & $29(12.2)$ & NS \\
\hline \multicolumn{5}{|l|}{ Time since initiation of ongoing therapy (months) } \\
\hline Chemotherapy & $n=516$ & $n=366$ & $n=150$ & \\
\hline Median (range) & $8.0(0-73.0)$ & $8.0(0-73.0)$ & $7.8(0-50.0)$ & NS \\
\hline Hormonotherapy & $n=68$ & $n=45$ & $n=23$ & \\
\hline Median (range) & $15.8(0-92.0)$ & $18.4(0-92.0)$ & $6.0(0-24.0)$ & $P<0.05$ \\
\hline Targeted therapy & $n=293$ & $n=187$ & $n=106$ & \\
\hline Median (range) & $12.9(0-73.0)$ & $13.4(0-73.0)$ & $12.0(0-57.0)$ & NS \\
\hline Immunotherapy & $n=117$ & $n=88$ & $n=29$ & \\
\hline Median (range) & $6.7(0-36.0)$ & $6.8(0-36.0)$ & $6.7(0-23.0)$ & NS \\
\hline PS score, n (\%) & $n=766$ & $n=529$ & $n=237$ & \\
\hline 0 & $252(32.9)$ & $157(29.7)$ & $95(40.1)$ & $P<0.01$ \\
\hline 1 & $353(46.1)$ & $250(47.3)$ & $103(43.5)$ & NS \\
\hline 2 & $116(15.1)$ & $91(17.2)$ & $25(10.5)$ & $P=0.02$ \\
\hline 3 & $27(3.5)$ & $19(3.6)$ & $8(3.4)$ & NS \\
\hline
\end{tabular}


Table 1 (continued)

\begin{tabular}{lllll}
\hline & Total patients & Low muscle mass & Unimpaired muscle mass & $P$ \\
\hline Non-responses & $18(2.3)$ & $12(2.3)$ & $6(2.5)$ & NS \\
\hline
\end{tabular}

$54.3 \%$ (94/173) in breast cancer patients; prevalence in the kidney, colon, and lung cancer sub-groups was comparable to that of the total population $(76.7 \%, 70.4 \%$, and $70.8 \%$, respectively). Patients with low muscle mass were twice more likely to have brain metastasis than their counterparts $(12.7 \%$ versus $5.9 \%, P<0.01)$. PS scores were poorer for patients with low muscle mass: $29.7 \%$ had a "good" PS score versus $40.1 \%$ without low muscle mass (PS $0, P<0.01$ ); $17.2 \%$ of low muscle mass patients had a "poor" PS score of 2 , compared to $10.5 \%$ of patients without low muscle mass $(P=0.02)$.

No significant difference was observed in the distribution of patients with and without low skeletal muscle mass by line of treatment and in the median time since primary cancer diagnosis. A majority of patients were receiving chemotherapy at the time of the study $(69.2 \%)$, followed by targeted therapy (39.8\%), immunotherapy (15.4\%), and hormonotherapy (9.4\%).

\section{Malnutrition and cachexia prevalence among cancer patients with low muscle mass}

Nutrition-related data are summarized in Table 2. About four times the proportion of patients without low muscle mass were obese compared to the low muscle mass group. Food intake during the previous meal, evaluated with a VAS, revealed that $9.5 \%$ of low muscle mass versus $5.1 \%$ of unimpaired muscle mass patients had very abnormal food intake (scores $0-4, P=0.04$ ) and $9.8 \%$ of low muscle mass patients versus $2.5 \%$ of their counterparts were moderately malnourished $(P<0.01)$. Serum albumin and GPS were found to be similar between both groups; nevertheless, CRP levels were significantly elevated in low muscle mass patients (mean $29.7 \pm 48.8 \mathrm{mg} / \mathrm{mL}$ versus $17.9 \pm 31.3 \mathrm{mg}$ / $\mathrm{mL}$ in patients with unimpaired muscle mass, $P<0.01)$. Cachexia prevalence in the sample was estimated at $38.4 \%$ (294/766 patients) for all three definitions by Fearon et al. [3]. Figure 2 represents the Venn diagram of the distribution of cachectic patients according to the definition to which they correspond.

\section{Multivariate analysis of the determinants of low muscle mass in cancer patients}

Only patients for whom serum albumin data were available $(n=561)$ were included in the multivariate analysis, as this was an exploratory variable (see the "Methods" section). Associations were found between low muscle mass and moderately malnourished status (OR 5.28, 95\% CI 1.74-16.00, $P<0.01$ ), severely malnourished status (OR $5.00,95 \%$ CI $1.05-23.82, P=0.04)$, and age $\geq 70$ years (OR 2.13, 1.27-3.56, $P<0.01$ ). Females (OR 0.23, 95\%CI $0.14-0.38, P<0.01)$ and those not having a brain metastatic site (OR $0.26,0.11-0.58, P<0.01)$ were found to have a lower risk of low muscle mass (Table $\mathrm{S} 1$ ).

\section{Low muscle mass diagnosis and management in real-life practice}

Figure 3 presents the level of disagreement between low muscle mass diagnosis via SMI measurement and the oncologist's assessment of low muscle mass. Whereas underestimation by physicians in non-obese patients with low muscle mass (44.9\%) was similar to that found in the total population, non-diagnosis in obese cancer patients was much higher, reaching $74.3 \%(P<0.01)$. Over a third of patients with low muscle mass were receiving personalized dietetic counselling compared to $24.1 \%$ of patients with unimpaired muscle mass $(P<0.01)$. Furthermore, a higher proportion of patients with low muscle mass (15.9\%) had future nutritional counselling planned by their oncologist, compared to those with unimpaired muscle mass $(6.1 \%, P<0.01)$. Nutritional management was available to $28.4 \%$ of patients with low skeletal muscle mass versus those without $(13.9 \%, P<0.01)$; $15.9 \%$ of all patients in the study were availing from physiotherapy, with no significant differences between patients with and without low muscle mass. Mean patient-reported daily spent-in-bed time was also higher for low muscle mass patients $(10.3 \pm 3.3 \mathrm{~h} /$ day $)$ than those without low muscle mass $(9.3 \pm 2.9 \mathrm{~h} /$ day, $P<0.01)$ (Table 3$)$.

\section{Impact of low muscle mass on ongoing cancer therapy tolerability}

Dose reductions, treatment interruptions, and occurrence of $\mathrm{AE}$ of grade 3 and greater related to ongoing treatment were comparable between low muscle mass patients and their counterparts. However, a higher number of low muscle mass patients (11.9\%) experienced treatment delays due to toxicities, compared to patients without low muscle mass $(6.8 \%$, $P=0.04$ ). Low muscle mass patients were also significantly more likely to have experienced at least two treatmentrelated AEs (13.8\% versus $8.4 \%$ among their counterparts; $P=0.03$ ) (Table S2). 
Table 2 Nutritional status, body weight measures, and Glasgow prognostic score (GPS in metastatic cancer patients with and without low skeletal muscle mass. Results are presented as $N(\%)$ or mean \pm SD. $N S$ not significant

\begin{tabular}{|c|c|c|c|c|}
\hline & Total patients & Low muscle mass & Unimpaired muscle mass & $P$ \\
\hline & $n=722$ & $n=500$ & $n=222$ & \\
\hline \multirow[t]{2}{*}{ Weight before cancer diagnosis $(\mathrm{kg})-$ mean $\pm \mathrm{SD}$} & $73.7 \pm 16.3$ & $73.4 \pm 15.4$ & $74.2 \pm 18.2$ & NS \\
\hline & $n=760$ & $n=525$ & $n=235$ & \\
\hline \multirow[t]{2}{*}{ Current weight (kg) } & $69.2 \pm 15.4$ & $68.1 \pm 14.5$ & $71.6 \pm 17.1$ & $P<0.01$ \\
\hline & $n=766$ & $n=529$ & $n=237$ & \\
\hline$>2 \%$ weight loss in 1 month & $157(20.5)$ & $120(22.7)$ & $37(15.6)$ & $P=0.02$ \\
\hline$>5 \%$ weight loss in 1 month & $59(7.7)$ & $46(8.7)$ & $13(5.5)$ & NS \\
\hline$>5 \%$ weight loss in 6 months & $217(28.3)$ & $164(31.0)$ & $53(22.4)$ & $P=0.01$ \\
\hline$>10 \%$ weight loss in 6 months & $93(12.1)$ & $74(14.0)$ & $19(8.0)$ & $P=0.03$ \\
\hline Current BMI $\left(\mathrm{kg} / \mathrm{m}^{2}\right)^{\mathrm{a}}$ & $n=766$ & $n=529$ & $n=237$ & \\
\hline Severely malnourished & $20(2.6)$ & $17(3.2)$ & $3(1.3)$ & NS \\
\hline Moderately malnourished & $58(7.6)$ & $52(9.8)$ & $6(2.5)$ & $P<0.01$ \\
\hline Non-responses & $10(1.3)$ & $6(1.1)$ & $4(1.6)$ & NS \\
\hline \multirow[t]{2}{*}{ Obese $\left(\mathrm{BMI} \geq 30 \mathrm{~kg} / \mathrm{m}^{2}\right)$} & $96(12.5)$ & $35(6.6)$ & $61(25.7)$ & $P<0.01$ \\
\hline & $n=760$ & $n=525$ & $n=235$ & \\
\hline Mean $\pm \mathrm{SD}$ & $24.6 \pm 4.6$ & $23.7 \pm 4.1$ & $26.6 \pm 5.0$ & $P<0.01$ \\
\hline VAS for food intake at latest meal (by the patient) ${ }^{b}$ & $n=766$ & $n=529$ & $n=237$ & \\
\hline $0-4$ very poor & $62(8.1)$ & $50(9.5)$ & $12(5.1)$ & $P=0.04$ \\
\hline $5-7$ poor & $199(26.0)$ & $146(27.6)$ & $53(22.64)$ & NS \\
\hline $8-10$ normal & $481(62.8)$ & $316(59.7)$ & $165(69.6)$ & $P<0.01$ \\
\hline Serum albumin $(\mathrm{SA}, \mathrm{g} / \mathrm{l})^{\mathrm{a}}$ & $n=766$ & $n=529$ & $n=237$ & \\
\hline Normal & $476(62.1)$ & $329(62.2)$ & $147(62.0)$ & NS \\
\hline Moderate malnutrition & $59(7.7)$ & $45(8.5)$ & $14(5.9)$ & NS \\
\hline Severe malnutrition & $26(3.4)$ & $19(3.6)$ & $7(3.0)$ & NS \\
\hline \multirow[t]{2}{*}{ Non-responses } & $205(26.8)$ & $136(25.7)$ & $69(29.1)$ & NS \\
\hline & $n=564$ & $n=395$ & $n=169$ & \\
\hline Mean \pm SD & $37.2 \pm 6.0$ & $37.2 \pm 5.9$ & $37.0 \pm 6.2$ & NS \\
\hline C-reactive protein $(\mathrm{mg} / \mathrm{l})$ & $n=381$ & $n=273$ & $n=111$ & \\
\hline Median (range) & $7.3(0-374.0)$ & $7.9(0-374.0)$ & $6.3(0-177.0)$ & $N S$ \\
\hline Glasgow prognostic score (GPS) & $n=348$ & $n=249$ & $n=99$ & \\
\hline 0 & $166(47.7)$ & $113(45.4)$ & $53(53.5)$ & NS \\
\hline 1 & 103 (29.6) & $75(30.1)$ & $28(28.3)$ & NS \\
\hline 2 & 79 (22.7) & $61(24.5)$ & $18(18.2)$ & NS \\
\hline
\end{tabular}

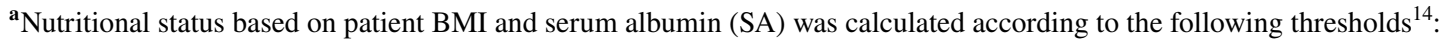

Moderate malnutrition

$-<70$ years old: $\mathrm{BMI} \leq 18.5$ to $16 \mathrm{~kg} / \mathrm{m}^{2}$ or $\mathrm{SA}<30$ to $20 \mathrm{~g} / \mathrm{L}$

- $\geq 70$ years old: $\mathrm{BMI}<21$ to $18 \mathrm{~kg} / \mathrm{m}^{2}$ or $\mathrm{SA}<35$ to $30 \mathrm{~g} / \mathrm{L}$

\section{Severe malnutrition}

$-<70$ years old: $\mathrm{BMI} \leq 16 \mathrm{~kg} / \mathrm{m}^{2}$ or $\mathrm{SA}<20 \mathrm{~g} / \mathrm{L}$

- $\geq 70$ years old: $\mathrm{BMI}<18 \mathrm{~kg} / \mathrm{m}^{2}$ or $\mathrm{SA}<30 \mathrm{~g} / \mathrm{L}$

${ }^{\text {b}}$ VAS: 0 (no food intake)-10 (normal food intake)

\section{Discussion}

Our prospective study represents the largest multicenter sample of metastatic cancer patients collected in France, assessing low muscle mass prevalence among 766 patients. As previously reported with different cut-offs for SMI [24,
25], low muscle mass was found to be highly prevalent among these cancer patients $(69.1 \%)$. These data demonstrate how commonly available CT imagery may be used to objectively measure muscle loss, thus facilitating diagnosis and subsequent treatment of this common condition in cancer patients. 
Fig. 2 Number and proportion of cachectic patients as per the three definitions of cachexia in patients with low skeletal muscle mass $(n=529)$

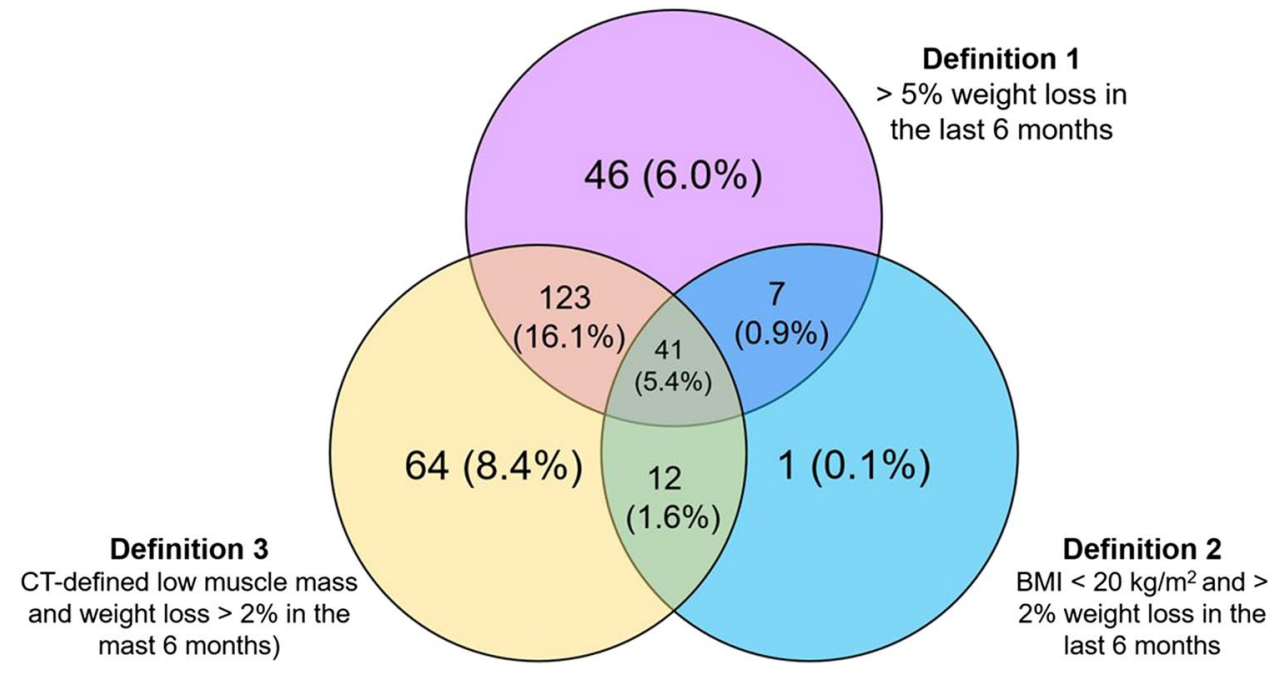

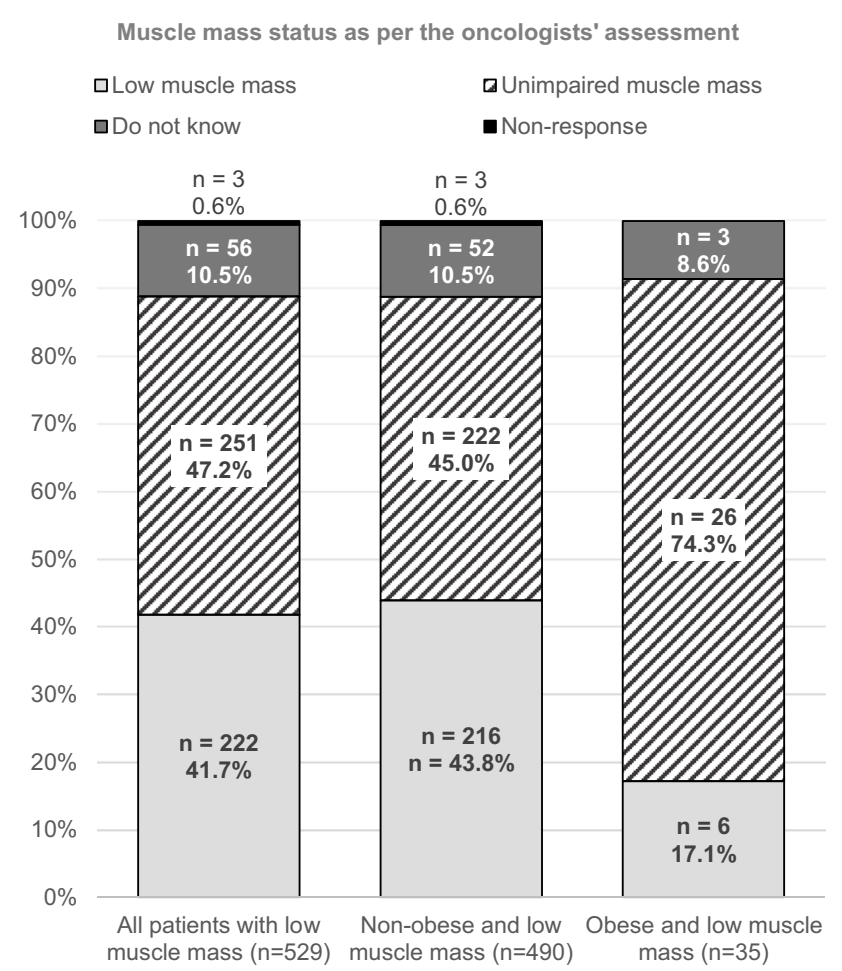

Patients with CT-scan defined low muscle mass, as determined via the SMI at the L3 level

Fig. 3 Skeletal muscle mass status as per the oncologists' assessment. Oncologists' diagnosis of low skeletal muscle mass is superposed to the L3 scan method using a stacked bar graph for the total low muscle mass population and for low muscle mass non-obese and obese sub-populations. Concordant and non-concordant oncologist assessments were calculated as a percentage of the low muscle mass population (y-axis); 249 of 529 patients with low skeletal muscle mass (47.1\%) were incorrectly evaluated as having low muscle mass by their oncologist; 220 of 490 (44.9\%) of non-obese low muscle mass patients were incorrectly evaluated. Among obese patients, the discordance was much higher, with 26 out of 35 (74.3\%) of low muscle mass patients being erroneously assessed as not having low skeletal muscle mass
We found that low muscle mass was significantly more prevalent in males than in females. Multivariate analyses also confirmed a strong association between low muscle mass prevalence and sex, with females being at lower risk. A similar sex-based association has been previously reported in other cancer sarcopenia populations [26-28]. In this study, low muscle mass prevalence was found to be highest in prostate cancer (94.4\%) and lower in breast cancer patients (54.3\%). Indeed, prostate cancer patients have multiple risk factors for muscle mass loss, such as advanced age, advanced cancer, and testosterone deprivation. In our study, prostate cancer patients had a mean age of $70.6 \pm 8.5$ versus $60.4 \pm 13.4$ years in breast cancer patients. Among sex-neutral cancers in this study, low skeletal muscle mass was similarly prevalent in each sex. Multivariate analysis did not reveal any associations between cancer type and the presence of low muscle mass.

The choice of the cut-off may be another factor explaining the differences in low muscle mass prevalence by sex. In studies where notably different SMI cut-off values were used, no significant differences in low muscle mass prevalence by sex was identified, such as in a recent study by Martin et al. [29], where L3 SMI thresholds for low muscular mass were stratified by BMI and sex, as can be seen when comparing the cut-off value that was employed in our work with those from 2 other studies based in Western populations $[25,26]$. In all cases, a very high prevalence of low muscle mass was found in this French population ( $>58 \%$; Table S3).

A 2016 literature review reported a prevalence of low muscle mass ranging from 5 to $89 \%$, depending on the malignancy and methods used for low muscle mass diagnosis [30]. Elsewhere, a prevalence of $25-30 \%$ has been reported, equivalent to about 1 million cancer patients in Europe [31]. There could also be other confounding factors influencing the apparent differences in low muscle mass prevalence by sex, such as biological and behavioral 
Table 3 Nutritional management and physical activity interventions in metastatic cancer patients, with and without low skeletal muscle mass. Unknown responses and non-responses are not reported in the table

\begin{tabular}{|c|c|c|c|c|}
\hline & Total patients & Low muscle mass & $\begin{array}{l}\text { Unimpaired } \\
\text { muscle mass }\end{array}$ & $P$ \\
\hline $\begin{array}{l}\text { Patients currently receiving personalized nutrition counselling from a } \\
\text { nutritionist, dietician or healthcare professional }\end{array}$ & $n=766$ & $n=529$ & $n=237$ & \\
\hline Yes & 237 (30.9) & $180(34.0)$ & $57(24.1)$ & $P<0.01$ \\
\hline No & $527(68.8)$ & $347(65.6)$ & $180(75.9)$ & \\
\hline If no, is any personalized nutritional counselling planned? & $n=527$ & $n=347$ & $n=180$ & \\
\hline Yes & $66(12.5)$ & $55(15.9)$ & $11(6.1)$ & $P<0.01$ \\
\hline Patients currently under specific nutritional management & $n=766$ & $n=529$ & $n=237$ & \\
\hline Yes & $183(23.9)$ & $150(28.4)$ & $33(13.9)$ & $P<0.01$ \\
\hline Oral nutritional supplements & $172(22.5)$ & $140(26.5)$ & $32(13.5)$ & $p<0.01$ \\
\hline Enteral nutrition & $6(0.8)$ & $6(1.1)$ & $0(0.0)$ & NS \\
\hline Parenteral nutrition & $7(0.9)$ & $6(1.1)$ & $1(0.4)$ & NS \\
\hline No & $580(75.7)$ & $377(71.3)$ & $203(85.7)$ & $P<0.01$ \\
\hline If $\mathrm{NO}$, is any personalized nutritional management planned? & $n=580$ & $n=377$ & $n=203$ & \\
\hline Yes & $56(9.7)$ & $44(11.7)$ & $12(5.9)$ & $P=0.05$ \\
\hline \multicolumn{5}{|l|}{ Current patient physical activity ${ }^{a}$} \\
\hline Number of hours spent in bed per day (hours/day) ${ }^{a}$ & $n=591$ & $n=412$ & $n=179$ & \\
\hline Mean \pm SD & $10.0 \pm 3.2$ & $10.3 \pm 3.3$ & $9.3 \pm 2.9$ & $P<0.01$ \\
\hline$\geq 11 \mathrm{~h}$ bedridden per day & $185(31.2)$ & $141(34.2)$ & $44(24.6)$ & $P<0.05$ \\
\hline Patients practicing physical exercises & $n=628$ & $n=429$ & $n=199$ & \\
\hline \multirow[t]{2}{*}{ Frequency of 30-min physical exercises per week ${ }^{\mathrm{b}}$ [median (range)] } & $1.2(0-35)$ & $1.1(0-28)$ & $1.4(0-35)$ & NS \\
\hline & $n=766$ & $n=529$ & $n=237$ & \\
\hline Patients undertaking physiotherapy & $122(15.9)$ & $86(16.3)$ & $36(15.2)$ & NS \\
\hline Patients consulting a sports coach & $22(2.9)$ & $12(2.3)$ & $10(4.2)$ & NS \\
\hline
\end{tabular}

ancluding sleeping time

${ }^{\mathrm{b}}$ Equivalent to $30 \mathrm{~min}$ of walking or cycling on flat terrain, or swimming

characteristics (hormones, immune responses, smoking, alcoholism, food habits). Nevertheless, an analysis that accounts for confounding factors was beyond the scope of our study, which is not longitudinal and was intended to be descriptive.

Our study is the first to demonstrate a potential association between brain metastases and low muscle mass. Patients with cerebral metastases are often prescribed corticosteroids, resulting in greater fatigue, and they also are at risk of developing hemiplegia. Both factors may lead to an overall reduction in the patients' physical activity, increasing the risk of low muscle mass.

Over 8 out of 10 cachectic patients had CT-defined low muscle mass (240 of 294 cachectic patients). This finding thus vouches for the use of CT imagery to diagnose low muscle mass, thereby promising a more reliable detection of cachexia.

The most worrying result of our study is that despite its high prevalence, practitioners were unable to recognize low skeletal muscle mass in nearly half of those patients with low muscle mass during the survey, even while completing nutritional status data. Low muscle mass was more poorly recognized in obese patients (Fig. 3). Our findings also demonstrate the general lack of nutritional and physical therapy support available to cancer patients in France (Table 3), as not more than a third of patients with low muscle mass were receiving nutritional counselling or nutritional management (in the form of supplements, enteral or parenteral nutrition). Less than 1 out of 5 patients with low muscle mass were undergoing physiotherapy. Low muscle mass patients have a higher risk of being bedridden for longer durations and having abnormal food intake. It is well known that suitable nutritional counselling and management and physical exercise regimes are helpful countermeasures to prevent muscle wasting, and hence cachexia development $[1,32]$.

These observations on nutritional support are sadly not new, as highlighted in the cross-sectional NutriCancer2012 study $[33,34]$ that evaluated malnutrition prevalence in over 2000 cancer patients in France. Malnutrition prevalence was around $40 \%$, and it was often diagnosed belatedly. Approximately $10 \%$ of these patients lacked any type of nutritional management, and there was only $70 \%$ concordance between the patients' true conditions and the physicians' evaluations. The study explained that such a low recourse to nutritional 
management was due to a lack of knowledge on malnutrition diagnosis and nutritional treatments (64\% of interviewed physicians) and the lack of a nutrition team in the hospital (56\% of physicians) [35]. While we could describe the existing nutrition and physical activity trends in patients with and without low muscle mass as part of this study, a longitudinal study would be better suited to investigate the link between these factors, their development and progression.

Low muscle mass patients have been reported to be more likely to accumulate treatment-related events [36-38]. In our study, however, no links could be established with dose reductions, treatment interruptions, and occurrence of grade 3 AEs. We did find an effect of low muscle mass on increased delays in treatment administration due to treatment-related toxicities. We suggest that our study design was such that it favored the selection of patients with low toxicity prevalence. This might also be explained by the relative heterogeneity in our sample with regard to ongoing treatment and cancer type (compared to other studies assessing low muscle mass and treatment toxicity [36-38]). However, the principal objective of this study was not to assess such toxicity, but to evaluate low muscle mass prevalence in a population of patients with metastatic cancers most frequently encountered in clinical settings.

We aimed to demonstrate the prevalence and impact of low muscle mass in a population of metastatic cancer patients in various healthcare settings in France. Measures taken to ensure data quality included the study's multicentric design, large sample size, oncologist and radiologist training in data collection, and collaboration with a coordinating center. However, some limitations were duly noted. This was a cross-sectional study that evaluated a patient's low muscle mass status at a certain timepoint, using their medical records to obtain much of the historical data reported. Such a study design offers limited control over center-based variability in the level of completeness and methods of measuring and recording of past data. Additionally, a cross-sectional design is inadequate for obtaining data on the development and evolution of cancer-related low muscle mass over the course of a person's illness, the evaluation of contributing factors, and its eventual impact on patient outcomes. Future longitudinal prospective studies, with reasonable follow-up durations, will be required to better understand this complex disorder.

It is possible that using cut-offs defined in a Canadian population could lead to an overestimated prevalence of low muscle mass in French patients, due to differences in population body weight demographics. Therefore, the use of the Canadian thresholds to evaluate the prevalence of muscle mass loss is one of the limitations of our study. Although the thresholds referred to in this study have been widely accepted in the literature, they are based on North American populations. This could lead to the risk of over- or under-estimations of prevalence when applied to our European study population. It has been established, for example, that cut-offs used for Western populations differ from those used for East Asian populations due to different morphotypes [39]. France and Canada, however, both represent Western populations and Martins et al. [14] have weighted their calculations using BMI (Table S3) to help account for different rates of obesity between these populations. A histogram depicting the SMI distribution among males and females in the SCAN study (Figure S1) shows that the mean SMI for females is $39.7 \mathrm{~cm}^{2} / \mathrm{m}^{2}$ and the mean SMI for males is $47.1 \mathrm{~cm}^{2} / \mathrm{m}^{2}$.

Since this study was performed across 29 centers, where different software was used to determine SMI, the inter-center precision of CT readings must be addressed. Recent studies have shown that CSA measurements may vary along with CT acquisition parameters; nevertheless, these variabilities would be clinically insignificant for the assessment low muscle mass $[40,41]$. Additionally, the decision to allow the use of different post-treatment stations (involving the extraction of measurements within the established threshold of -29 to $+150 \mathrm{HU}$ followed by manual segmentation) was supported by data from a study that compared four dedicated post-treatment stations and demonstrated an excellent correlation between readings, with very limited inter- and intra-operator variability [18]. Moreover, the effect of contrast media and varying slice thickness on differences in body composition assessments had to be considered [16, 42], which is why mandatory distance training was provided to the investigating radiologists to standardize these measurements as much as possible. If the objective of the study had been a longitudinal monitoring of muscle mass changes, the type of CT parameters, post-treatment stations, contrast media, and slice thickness choices would have been standardized across centers. However, for a cross-sectional study seeking to describe the real-life prevalence of low skeletal muscle mass, these limitations were considered acceptable for the purpose of the investigation.

Another methodological limitation is the lack of standardization (and possibly even homogeneity) of the oncologists' chosen methods of low muscle mass diagnosis, which limited our ability to compare the accuracy of their diagnosis relative to CT-defined low muscle mass. Since there is no standard method of evaluating low muscle mass in cancer patients in France, oncologists were permitted to assess this as per their knowledge and experience. The results of this descriptive analysis should therefore be interpreted with caution.

\section{Conclusions}

Low muscle mass was found to be highly prevalent, at $69.1 \%$, in patients with metastatic cancers in French clinical practice as evaluated by L3 level CT of muscle mass measurements. 
Nutritional and physical therapy support were only provided to a minority of patients with low skeletal muscle mass. Low muscle mass was often underdiagnosed by the oncologist, even more so in obese patients, highlighting a worrying gap in the oncologists' understanding of muscle wasting diagnosis and management. Our findings underline the necessity for improvements in oncologist education of diagnostic tools and the nutritional aspects of cancer management, and the need for an increased involvement of radiologists, dieticians, nutritionists, physiotherapists, and fitness counsellors in the regular, long-term management of cancer-related low muscle mass as part of a more wholistic standard of care.

Supplementary Information The online version contains supplementary material available at https://doi.org/10.1007/s00520-021-06603-0.

Author contribution B. Raynard, F. Pigneur, M. Di Palma, E. Deluche, and F. Goldwasser equally contributed to the conception and design of the research; B. Raynard, F. Pigneur, M. Di Palma, E. Deluche, and F. Goldwasser equally contributed to the acquisition and analysis of the data; B. Raynard contributed to the interpretation of the data; and B. Raynard drafted the manuscript. All authors critically revised the manuscript, agree to be fully accountable for ensuring the integrity and accuracy of the work, and read and approved the final manuscript.

Funding Fresenius Kabi France sponsored this study.

Data and materials availability N/A.

Code availability N/A.

\section{Declarations}

Ethics approval This study, conducted in accordance with the Declaration of Helsinki, was approved by the French ethical research committee, "Le Comité de Protection des Personnes" (CPP), on 6 July 2017 (approval no. 2017-A01648-45). National data protection regulations were also met (CNIL, approval no. 2066086).

Consent to participate All persons provided their informed consent prior to enrollment.

Consent for publication All persons were informed that this study would be submitted for publication.

Conflict of interest All authors were remunerated for their participation as investigators in the SCAN study.

Open Access This article is licensed under a Creative Commons Attribution 4.0 International License, which permits use, sharing, adaptation, distribution and reproduction in any medium or format, as long as you give appropriate credit to the original author(s) and the source, provide a link to the Creative Commons licence, and indicate if changes were made. The images or other third party material in this article are included in the article's Creative Commons licence, unless indicated otherwise in a credit line to the material. If material is not included in the article's Creative Commons licence and your intended use is not permitted by statutory regulation or exceeds the permitted use, you will need to obtain permission directly from the copyright holder. To view a copy of this licence, visit http://creativecommons.org/licenses/by/4.0/.

\section{References}

1. Aoyagi $\mathrm{T}$ et al (2015) Cancer cachexia, mechanism and treatment. World J Gastrointest Oncol 7(4):17-29

2. Fearon K, Arends J, Baracos V (2013) Understanding the mechanisms and treatment options in cancer cachexia. Nat Rev Clin Oncol 10(2):90-99

3. Fearon K et al (2011) Definition and classification of cancer cachexia: an international consensus. Lancet Oncol 12(5):489-495

4. Chemama S, Raynard B, Antoun S (2016) Impact of cancer muscle mass loss on anticancer treatment toxicities. Bull Cancer 103(9):786-793

5. Khan $\mathrm{S}$ et al (2016) Detection and evaluation of malnutrition in oncology: what tools, what type of cancer and for what purposes? Bull Cancer 103(9):776-785

6. Melchior JC et al (2012) Clinical and economic impact of malnutrition per se on the postoperative course of colorectal cancer patients. Clin Nutr 31(6):896-902

7. Senesse P et al (2012) Nutrition chez le patient adulte atteint de cancer: Recommandations professionnelles de la Société Francophone Nutrition Clinique et Métabolisme (SFNEP)

8. Coin A et al (2013) Prevalence of sarcopenia based on different diagnostic criteria using DEXA and appendicular skeletal muscle mass reference values in an Italian population aged 20 to 80. J Am Med Dir Assoc 14(7):507-512

9. Cruz-Jentoft AJ et al (2010) Sarcopenia: European consensus on definition and diagnosis: report of the European Working Group on Sarcopenia in Older People. Age Ageing 39(4):412-423

10. Cruz-Jentoft AJ et al (2019) Sarcopenia: revised European consensus on definition and diagnosis. Age Ageing 48(1):16-31

11. Lieffers JR et al (2012) Sarcopenia is associated with postoperative infection and delayed recovery from colorectal cancer resection surgery. Br J Cancer 107(6):931-936

12. Prado CM et al (2007) Body composition as an independent determinant of 5-fluorouracil-based chemotherapy toxicity. Clin Cancer Res 13(11):3264-3268

13. $\mathrm{Su} \mathrm{H}$ et al (2019) CT-assessed sarcopenia is a predictive factor for both long-term and short-term outcomes in gastrointestinal oncology patients: a systematic review and meta-analysis. Cancer Imaging 19(1):82

14. Martin L et al (2020) Cancer-associated malnutrition and CTdefined sarcopenia and myosteatosis are endemic in overweight and obese patients. JPEN J Parenter Enteral Nutr 44(2):227-238

15. Biolo G, Cederholm T, Muscaritoli M (2014) Muscle contractile and metabolic dysfunction is a common feature of sarcopenia of aging and chronic diseases: from sarcopenic obesity to cachexia. Clin Nutr 33(5):737-748

16. Mourtzakis M et al (2008) A practical and precise approach to quantification of body composition in cancer patients using computed tomography images acquired during routine care. Appl Physiol Nutr Metab 33(5):997-1006

17. Perthen JE et al (2018) Intra- and interobserver variability in skeletal muscle measurements using computed tomography images. Eur J Radiol 109:142-146

18. van Vugt JL et al (2017) A comparative study of software programmes for cross-sectional skeletal muscle and adipose tissue measurements on abdominal computed tomography scans of rectal cancer patients. J Cachexia Sarcopenia Muscle $8(2): 285-297$

19. Prado CM et al (2008) Prevalence and clinical implications of sarcopenic obesity in patients with solid tumours of the respiratory and gastrointestinal tracts: a population-based study. Lancet Oncol 9(7):629-635 
20. Thibault $\mathrm{R}$ et al (2009) Use of 10-point analogue scales to estimate dietary intake: a prospective study in patients nutritionally at-risk. Clin Nutr 28(2):134-140

21. Oken MM et al (1982) Toxicity and response criteria of the Eastern Cooperative Oncology Group. Am J Clin Oncol 5(6):649-655

22. HAS (2019) Diagnosis of malnutrition in children and adults: methodological recommendations for clinical practice

23. Forrest LM et al (2004) Comparison of an inflammation-based prognostic score (GPS) with performance status (ECOG) in patients receiving platinum-based chemotherapy for inoperable non-small-cell lung cancer. Br J Cancer 90(9):1704-1706

24. Pamoukdjian F et al (2018) Prevalence and predictive value of pre-therapeutic sarcopenia in cancer patients: a systematic review. Clin Nutr 37(4):1101-1113

25 Shachar SS, Williams G, Muss HB, Nishijima TF (2016) Prognostic value of sarcopenia in adults with solid tumours: a metaanalysis and systematic review. Eur J Cancer 57:58-67

26. Caan BJ, Meyerhardt J, Kroenke CH, Alexeeff S, Xiao J, Weltzien E, Feliciano EC, Castillo AL, Quesenberry CP, Kwan ML, Prado CM (2017) Explaining the obesity paradox: the association between body composition and colorectal cancer survival (C-SCANS Study). Cancer Epidemiol Biomark Prev. 26(7):1008-1015

27. Antoun S, Baracos V, Birdsell L, Escudier B, Sawyer MB (2010) Low body mass index and sarcopenia associated with dose-limiting toxicity of sorafenib in patients with renal cell carcinoma. Ann Oncol 21(8):1594-1598

28 Prado CM, Lieffers J, McCargar LJ, Reiman T, Sawyer MB, Martin L, Baracos VE (2008) Prevalence and clinical implications of sarcopenic obesity in patients with solid tumours of the respiratory and gastrointestinal tracts: a population-based study. Lancet Oncol 9(7):629-35

29. Martin L et al (2013) Cancer cachexia in the age of obesity: skeletal muscle depletion is a powerful prognostic factor, independent of body mass index. J Clin Oncol 31(12):1539-1547

30 Rier HN, Jager A, Sleijfer S, Maier AB, Levin M-D (2016) The prevalence and prognostic value of low muscle mass in cancer patients: a review of the literature. Oncology 21(11):1396-1409. https://doi.org/10.1634/theoncologist.2016-0066

31. von Haehling S, Anker MS, Anker SD (2016) Prevalence and clinical impact of cachexia in chronic illness in Europe, USA, and Japan: facts and numbers update 2016. J Cachexia Sarcopenia Muscle 7(5):507-509
32. Aapro $M$ et al (2014) Early recognition of malnutrition and cachexia in the cancer patient: a position paper of a European School of Oncology Task Force. Ann Oncol 25(8):1492-1499

33. Gyan E et al (2017) Malnutrition in patients with cancer. JPEN J Parenter Enteral Nutr 148607116688881

34 Lacau St Guily J et al (2018) NutriCancer: a French observational multicentre cross-sectional study of malnutrition in elderly patients with cancer. J Geriatr Oncol 9(1):74-80

35. Raynard B, Hébuterne X, Goldwasser F, AitHssain A, DubrayLongeras P, Barthélémy P, Rosso E, Phoutthasang V, Bories C, Digue L, Laharie D, Desport JC, Falkowski S, Lacau Saint Guily J, Gyan E (2017) Crossed perceptions about malnutrition in patients and their doctors in oncology. Bull Cancer. 104(11):921-928

36. Barret $\mathrm{M}$ et al (2014) Sarcopenia is linked to treatment toxicity in patients with metastatic colorectal cancer. Nutr Cancer 66(4):583-589

37. Ross PJ et al (2004) Do patients with weight loss have a worse outcome when undergoing chemotherapy for lung cancers? Br J Cancer 90(10): 1905-1911

38. Van Cutsem E, Arends J (2005) The causes and consequences of cancer-associated malnutrition. Eur J Oncol Nurs 9(Suppl 2):S51-63

39. Fujiwara $\mathrm{N}$ et al (2015) Sarcopenia, intramuscular fat deposition, and visceral adiposity independently predict the outcomes of hepatocellular carcinoma. J Hepatol 63(1):131-140

40. Paris MT et al (2018) Influence of contrast administration on computed tomography-based analysis of visceral adipose and skeletal muscle tissue in clear cell renal cell carcinoma. JPEN J Parenter Enteral Nutr 42(7):1148-1155

41. van Vugt JLA et al (2018) Contrast-enhancement influences skeletal muscle density, but not skeletal muscle mass, measurements on computed tomography. Clin Nutr 37(5):1707-1714

42. Morsbach F et al (2019) Body composition evaluation with computed tomography: Contrast media and slice thickness cause methodological errors. Nutrition 59:50-55

Publisher's note Springer Nature remains neutral with regard to jurisdictional claims in published maps and institutional affiliations. 